\title{
Biological transfer of radionuclides in marine environments - identifying and filling knowledge gaps for environmental impact assessments
}

\author{
J .E. Brown, P. Børretzen and A. Hosseini \\ Norwegian Radiation Protection Authority, Grini næringspark 13, Postbox 55, \\ 1332 Østerås, Norway
}

\begin{abstract}
Methodologies have been developed to allow impacts of ionising radiation on marine ecosystems to be evaluated within selected geographical settings. The stages in the assessment require initial information about unit concentrations of radionuclides in reference media to be collated. Activity concentrations for reference groups of flora and fauna and for representatives of these groups are then derived using an equilibrium concentration factor approach. Following this, dose-rates can be calculated using relevant dose conversion factors. Impacts on the environment are evaluated by comparison with dose-rates at which selected biological effects are known to occur and the natural radiation background. This paper focuses mainly on the transfer part of the assessment drawing attention to gaps in information that have been identified through review and providing an outline of methods that may be used to fill these gaps. Biokinetic models parameterised using allometric relationships have shown their utility in this respect. Initial work looking into the application of such models has met with some success with model predictions comparing favourably with the few empirical data available. Further work will be needed to validate the models for a large range of radionuclides and to consider uncertainty in model estimates through probabilistic analyses.
\end{abstract}

\section{ENVIRONMENTAL IMPACT ASSESSMENT}

Methodologies to assess the impact of exposure to ionising radiation on flora and fauna have been developed in two recent European collaborative projects [1-3]. The initial stage of the assessment requires the selection of appropriate reference biota and suitable representative organisms (normally defined at the species level) with concomitant collation of life history data sheets. Methods for deriving the transfer and fate of radionuclides are necessary during the assessment procedure as are methods for deriving (weighted or unweighted) dose-rates. Once exposures for reference biota have been derived, they need to be interpreted in terms of biological effects. The analyses cannot be termed a risk assessment at this stage because the probability of specified biological effects occurring has not been adequately considered.

\subsection{Reference organisms and Representative species}

The FASSET definition of "reference organism" is: "a series of entities that provides a basis for the estimation of the radiation dose-rate to a range of organisms that are typical, or representative, of a contaminated environment. These estimates, in turn, would provide a basis for assessing the likelihood and degree of radiation effects." [3]. For Arctic marine environments in the EPIC project, selection criteria have been applied in order to select a reference organism suite [4]. These were: 
- Ecological niche. This was simply applied as a requirement to have at least one representative from each trophic level.

- Intrinsic radiosensitivity. In this case comparison was made between the acute lethal doses expressed by various organism groups.

- Radioecological sensitivity, i.e. identification of which organisms are likely to be most exposed either through an expression of relatively high radionuclide bioaccumulation or relatively high activity concentrations in their habitat.

- Distribution. Preference was given to those organisms that were year-round residents in the Arctic.

- Amenability to research and monitoring. This criterion involved an assessment of whether data sets documenting activity concentrations in various groups of organism were available from monitoring studies and whether future research might be conducted upon the various groups (e.g. exposure experiments etc.).

The generic reference organism lists have been used as a basis for deriving appropriate environmental transfer data information and selecting suitable target geometries/phantoms for dosimetric modelling. With respect to these points, it became apparent that the identification of actual species (or in some cases families or classes of organisms) representing each of the broadly defined groups would be helpful in some instances. This was true in the case of deriving food-chain model parameters where detailed information was often required, beyond a generic consideration, with respect to organism characteristics. It was also true in the case of geometry construction where quantitative information on size, shape and density are required and can be derived, simply and transparently, from a consideration of real flora and fauna. Examples of suitable representative species of selected reference organisms were subsequently chosen giving preference to species ubiquitous throughout the specified assessment area, e.g. European Arctic, and the availability of appropriate data. Basic ecological information needs to be collated for each of the selected flora and fauna. The specific organism attributes that should be considered relate directly to the subsequent assessment of exposure. For example, information should be provided on habitat and, where applicable, the fractional occupancy of various organisms in their habitats. This information is important for the weighting of external dose-rates in order to account for the behaviour of the organism. Guidance on the types of ecological information required for reference fauna is provided elsewhere [2, 3]. The resultant initial reference organism list with representative types, in Arctic systems (see [5]), includes examples such as Fucus spp. for macroalgae, Boreogadus saida (polar cod) for Pelagic planktotrophic fish and 'Seals' (e.g. Phoca groenlandica) for carnivorous mammal.

\subsection{Dose calculation}

The whole-body absorbed dose-rate is used as a measure of the reference organism exposure to ionising radiation, expressed in units of Gy per unit time, and is the sum of internal and external absorbed dose-rates. It may be appropriate to introduce radiation weighting factors to take account of the differing biological effectiveness of different types of ionising radiation. At the present time such consideration is recommended for alpha particle radiation, and for beta particle radiation with mean particle energies less than $10 \mathrm{keV}$. Introduction of these weighting factors leads to the weighted absorbed dose-rates, for example:

$$
\begin{aligned}
& \dot{D}_{\text {total,weighted }}^{j}=\dot{D}_{\text {int, weighted }}^{j}+\dot{D}_{\text {ext, weighted }}^{j} \\
& \dot{D}_{\text {int, weighted }}^{j}=w_{\text {low } \beta} \dot{D}_{\text {int,low } \beta}^{j}+\dot{D}_{\text {int }, \beta \gamma}^{j}+w_{\alpha} \dot{D}_{\text {int }, \alpha}^{j}
\end{aligned}
$$

where $w_{\text {low } \beta}$ and $w_{\text {low }}$ are the radiation weighting factors for low energy beta radiation, and alpha radiation, respectively and the subscripts $l o w \beta, \beta \gamma$, and $\alpha$ denote the contributions to absorbed dose-rate from low energy beta particles, other beta particles and gamma ray photons, and alpha particles, respectively. 
Contributions from low energy beta particles and alpha particles to external radiation will usually be negligible, but may need to be considered for organisms whose dimensions are of the same order as the range of these radiation types in tissue - typically, in the sub-millimetre range.

The external (absorbed) dose-rate, averaged over different habitats, can be determined by the following equation:

$$
\dot{D}_{e x t}^{j}=\sum_{z} v_{z} \sum_{i} C_{z i}^{r e f} * D C F_{e x t, z i}^{j}
$$

where,

$v_{z}$ is the occupancy factor, i.e. fraction of the time that the organism $j$ expends at a specified location $\mathrm{z}$ in its habitat.

$C_{z i}{ }^{r e f}$ is the average concentration of the radionuclide $i$ in the reference media of a given location $\mathrm{z}$ (Bq kg-1 (soil or sediment) or $\mathrm{Bq} \mathrm{m}^{-3}$ (water)),

$D C F^{j}{ }_{\text {ext } z i}$ is the dose conversion factor for external exposure defined as the ratio between the average activity concentration of the radionuclide $i$ in the reference media corresponding to the location $z$ and the dose rate to the organism $j\left(\mathrm{~Gy} \mathrm{y}^{-1}\right.$ per $\mathrm{Bq} \mathrm{kg}^{-1}$ or $\mathrm{Bq} \mathrm{m}^{-3}$ ).

The internal (absorbed) dose-rate can be derived from the activity concentration in the selected reference organism using the following equation:

$$
\dot{D}_{\mathrm{int}}^{j}=\sum_{i} C_{i}^{j} * D C F_{\mathrm{int}, i}^{j}
$$

where,

$C_{i}^{j}$ is the average concentration of the radionuclide $i$ in the reference organism $j\left(\mathrm{~Bq} \mathrm{~kg}{ }^{-1}\right.$ fresh weight); $D C F_{\text {int }, i}^{j}$ is the radionuclide-specific dose conversion factor (DCF) for internal exposure defined as the ratio between the average concentration of the radionuclide $i$ in the organism $j$ and the dose-rate to the organism ( $\mathrm{Gy} \mathrm{a}{ }^{-1}$ per $\mathrm{Bq} \mathrm{kg}^{-1}$ fresh weight).

The derivation of internal and external DCFs for reference biota are discussed and radionuclide-specific values presented in numerical form in EPIC [6] and FASSET reports [7].

\subsection{Assessment of effects}

Large data collation exercises have been undertaken in both FASSET [8] and EPIC [9] projects for the purpose of evaluating doses to flora and fauna. The approach taken within EPIC [2] with regards to analyses of dose-effects relationships, was to collate and organise data around the reference organism categories and to focus on dose-rates and biological endpoints that are of relevance from the perspective of environmental protection. Data of dose-effects relationships on radiation effects in biota available from Russian and other former Soviet Union sources have been collated. The compiled data are concentrated on the effects in radiosensitive species in terrestrial and aquatic ecosystems, such as mammals, fish, and sensitive groups of plants (e.g. pines). Data have been organised under "umbrella" end-point categories, namely: morbidity, reproduction, mortality, cytogenetic effects, ecological effects, stimulation effects and adaptation effects. A general conclusion was made, that the threshold for deterministic radiation effects in wildlife lies somewhere in the range $0.5-1 \mathrm{mGy} \mathrm{d}^{-1}$ for chronic low-LET radiation. However, although minor effects on morbidity in sensitive vertebrate animals are observed at the dose range specified above, populations of highly productive vertebrate organisms are viable at dose rates in the order $10 \mathrm{mGy} \mathrm{d}^{-1}$. Preliminary scales defining the severity of radiation effects at different levels of chronic exposure for different organisms groups have been constructed. In addition, background dose-rates have been calculated for reference organisms in terrestrial, freshwater and marine ecosystems although some of the values generated have been based on very limited data sets. 


\section{BIOLOGICAL TRANSFER}

\subsection{Empirical review}

A review of the literature was conducted with the purpose of collating Concentration Factor (CF) information specifically for reference or representative flora and fauna in European [3] and Arctic [5] marine environments. Such information was considered to be a useful supplement to the extensive review undertaken by the IAEA [10]. In a review of CF data coverage in the FASSET project, few data were found for radionuclides such as I, Ru, Ra, Np, Cm and U no data were found for Th, C, H, $\mathrm{Nb}, \mathrm{Ni}$ and $\mathrm{Cl}$. No data for vascular plants were found. Marine birds, polycheate worms and mammals are particulary poorly characterised using CF datasets.

Due to the interplay of many factors, both biological and environmental, variance of CFs is great within and between studies. For example, Sazykina [11] found that the values of Cs-137 CF for fish (Barents Sea, cod) were not constant, but gradually changed from 28 \pm 5 in 1979 up to $182 \pm 48$ in 1992. This study clearly illustrates what is usually a major source of variation in many studies; lack of equilibrium within the period of observations. Consequently, caution must be used when an organism contamination is predicted by the radionuclide 'concentration factor' approach.

\subsection{Models to fill transfer data gaps}

The CF approach has been criticised for numerous reasons (see [12]) but in the construction of this methodology the fact that the uptake of many radionuclides to certain reference organism types are poorly, if at all, described were deemed particularly important. For this reason other approaches to deriving information on transfer to biota have been explored.

Biokinetic models may allow more realistic prognoses concerning the dynamic response of an ecological system to be made and allow tentative estimates to be derived concerning equilibrium CFs. Allometric relationships may provide surrogate values in cases where data are lacking on some of the parameters required for simulation. The allometric approach is based on the observation that many metabolic parameters, including basal metabolic rates, ingestion rates, biological half times, are proportional to the mass of an organism. Such approaches have been applied elsewhere for the very purpose of deriving transfer data where empirical data sets are unsatisfactory [13, 14]. To demonstrate how this type of model might be employed to fill the required knowledge gaps, a simple food chain model has been developed, based on information in the open literature [15], to consider the transfer of selected radionuclides $\left({ }^{137} \mathrm{Cs}\right.$ and $\left.{ }^{239,240} \mathrm{Pu}\right)$ to reference organisms in the pelagic food chain: phytoplankton-zooplankton-polar cod-harp seal. [16, 5].

The model, based on earlier work [17, 18], considers uptake via food and water for aquatic organisms. Excretion/elimination rates are considered to be independent of the uptake route, the assimilation efficiency is considered to be independent of food type, and the activity concentration in gut content of prey species is not considered to become assimilated by the predator. Further assumptions are that the phytoplankton and zooplankton (trophic levels 1 and 2) are considered as homogeneous groups described by specified parameter values rather than ranges and that the growth rate for all organisms is 0 . This last assumption may be a particularly poor one [17], but the complexity of the weight dynamics for the organisms in question will require more detailed further study. The sets of $1^{\text {st }}$ order differential equations that have been used to characterise the system and the parameterisation of the model have been described elsewhere [16]. Selected output from these model runs are presented in Figure 1. 


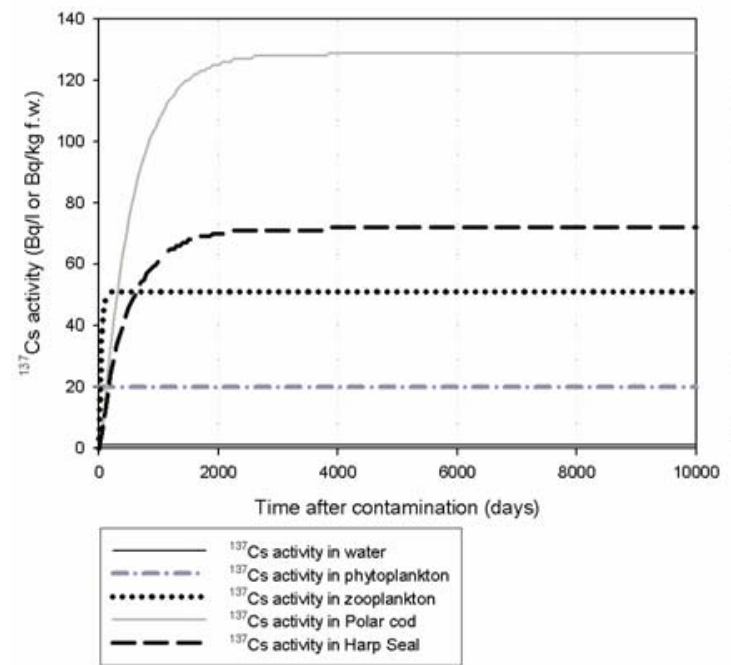

a)

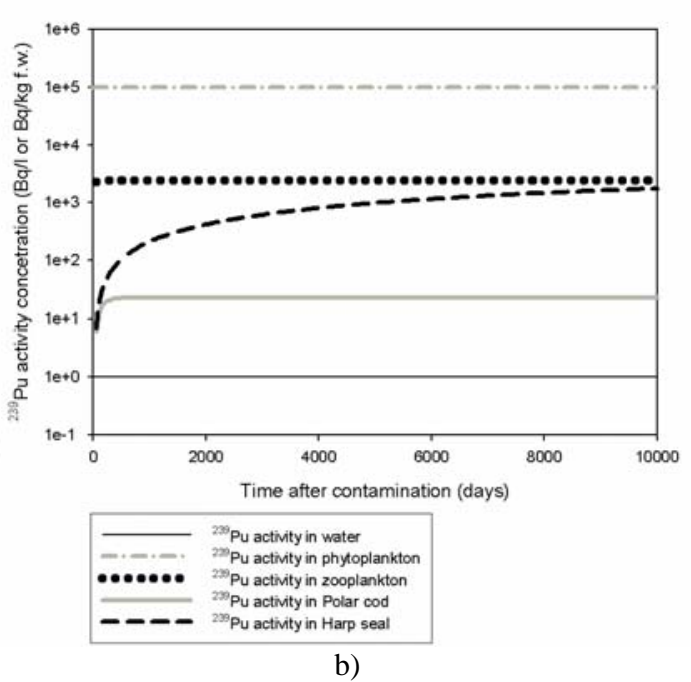

b)

Figure 1. Activity concentrations (fresh weight) of (a) ${ }^{137} \mathrm{Cs}$ and (b) ${ }^{239} \mathrm{Pu}$ for selected marine organisms derived from biokinetic allometric modelling. Results for Polar cod and seal are for whole body. Figures from [16].

For ${ }^{137} \mathrm{Cs}$ (Figure 1a), biomagnification (increase in body mass concentration of a contaminant as it passes from low trophic levels to higher ones) appears to occur for the lower trophic levels, but is not occurring at the highest trophic level i.e. seal. The results from model runs suggest that equilibrium is not attained for higher trophic levels - polar cod and harp seal -after initial contamination for 2000 days. This has obvious implications in relation to the interpretation of field data if activity concentrations in water are changing rapidly with time. Equilibrium ${ }^{137} \mathrm{Cs} \mathrm{CF}$ values are approximately 50, 130 and 70 for zooplankton, polar cod and seal, respectively. They compare well with IAEA values [10] recommended values of 40 for zooplankton and 100 for generic fish. The ${ }^{137} \mathrm{Cs}$ value of 70 for seal falls within the range reported by IAEA.

Transfer of ${ }^{239} \mathrm{Pu}$ (Figure 1b) to successively higher trophic levels is low, with a decrease of several orders of magnitude observed between primary producers, represented by phytoplankton, and polar cod representing trophic level 3-4. However, the model predicts that this decreasing trend in activity concentrations along the food-chain is reversed for the highest trophic level, represented by seal. Equilibrium is attained very slowly for seals (reflecting in part, the very low, allometrically-derived excretion rate). In this case, equilibrium is only truly obtained after $6 \times 10^{4}$ days (165 years) of simulation. Clearly, even in the unlikely circumstance where water concentrations remain unchanged over highly protracted time scales, equilibrium is unlikely to occur over the life-time (in the order of decades) of the seal.

The equilibrium Pu CF values of $2.5 \times 10^{3}$ (zooplankton) and 25 (polar cod) predicted from model runs, compare favourably with IAEA values [10] recommended values of $4 \times 10^{3}$ and 100 for zooplankton and generic fish, respectively. For seals, as discussed above, a true equilibrium CF value of $4.5 \times 10^{3}$ is not obtained between the water and body compartments over the life time of the organism. It therefore may be more appropriate to select a suitable "contact period" that reflects the in situ conditions. Using an arbitrarily selected, 5 year contact time, a concentration ratio value of circa 390 was predicted. This latter value compares well with the empirically-derived value presented in relevant reviews $[5,16]$ although it still differs substantially from the IAEA value. 


\section{CONCLUSIONS}

A methodology has been constructed allowing the transfer and doses of biota to be calculated and doses to be assessed in terms of natural background dose-rates and dose-rates at which observable biological effects are known to occur. With regards to the initial stage of the analyses information gaps relating to the transfer of radionucldies to some reference organism types have been noted. Biokinetic models, parametrised to some extent with the use of allometric information, appear to provide a useful tool for filling some of these gaps. Example simulations appear to provide sensible results for selected reference fauna CFs, agreeing well with empirically derived values. Furthermore the work draws attention to the fact that equilibrium is often not attained and that CFs, under the common understanding of the term, may not be directly applicable. Clearly the scope of such models should be extended to other radionuclides - notably work in this direction was conducted in the FASSET project - moreover any modelling output arising from such studies requires validation against empirical data sets. This would appear to require further laboratory based uptake studies and/or field studies. Uncertainty and variability within each stage of the impact assessment requires quantification. For the transfer stage of the assessment this may involve statistical analyses of all parameters used in the models applied.

\section{Acknowledgments}

The work discussed here has been conducted as part of the FASSET project, conducted under a contract (FIGE-CT-2000-00102) within the EC EURATOM research programme and the EPIC project conducted under a contract (ICA2-CT-2000-10032) within the EC Inco-Copernicus research programme. The support of the EC is gratefully acknowledged. The authors are also grateful to their Russian partners in the EPIC project SPA TYPHOON, Institute of Radiation Hygiene for making the EPIC project a success.

\section{References}

[1] Larsson, C-M., Strand, P. \& Brown, J.E. EPIC-FASSET. In: Proceeding from the International Conference on radioactivity in the environment, Monaco, 1-5 September 2002, P. Børretzen, T. Jølle \& P. Strand (eds.), (NRPA, Østerås, 2002) pp.39-42.

[2] Brown J.E., Thørring H. and Hosseini A., The "EPIC” Impact Assessment Framework - A Deliverable Report for EU Funded Project ICA2-CT-2000-10032 (Norwegian Radiation Protection Authority, Østerås, 2003) pp. 175.

[3] Brown, J.E., Strand, P., Hosseini, A. \& Børretzen, P. (Eds.). Handbook for assessment the exposure of biota to ionising radiation from radionuclides in the environment. Deliverable Report for the EC Project FASSET Contract No. FIGE-CT-2000-00102 (Norwegian Radiation Protection Authority, Østerås, Norway, 2003) pp.101.

[4] Beresford, N.A., Wright, S.M. \& Sazykina, T. (Eds.). Reference Arctic organisms. Deliverable Report 1 for EPIC. EC Inco-Copernicus project ICA2-CT-2000-10032. (CEH, Grange-over-Sands, UK, 2001).

[5] Beresford, N.A., Wright, S.M., Brown, J.E. \& Sazykina, T. (Eds.). Review of approaches for the estimation of radionuclide transfer to reference Arctic biota. Deliverable Report 3 for EPIC. EC Inco-Copernicus project ICA2-CT-2000-10032. (CEH, Grange-over-Sands, UK, 2003).

[6] Golikov, V. \& Brown, J.E. (Eds.). Internal and External Dose Models. Deliverable report 4 for EPIC. EC Inco-Copernicus project ICA2-CT-2000-10032. (Norwegian Radiation Protection Authority, Østerås, Norway, 2003) pp. 52.

[7] Pröhl G., Brown, J., Gomez-Ros, J.-M., Jones, S., Woodhead, D., Vives, J., Taranenko, V., Thørring, H. . Dosimetric models and data for assessing radiation exposure to biota. Deliverable 3 to the Project "FASSET" Framework for the assessment of Environmental Impact, contract No. FIGE-CT-2000-00102. (Swedish Radiation Protection Authority., Sweden, 2003). 
[8] Woodhead, D. \& Zinger, I.Radiation effects on plants and animals. FASSET Deliverable Report 4. Contract No FIGE-CT-2000-00102. (Norwegian Radiation Protection Authority, Østerås, 2003)

[9] Sazykina, T.G., Jaworska, A. \& Brown, J.E. (Eds.). Dose-effects relationships for reference (or related) Arctic biota. Deliverable Report 5 for the EPIC project Contract no. ICA2-CT-200-10032. (Norwegian Radiation Protection Authority, Østerås, 2003) pp. 119.

[10] International Atomic Energy Agency (IAEA). Sediment distribution coefficients and Concentration Factors for biota in the Marine Environment, Technical Reports Series No. 422, (International Atomic Energy Agency, Vienna, 2004).

[11] Sazykina, T.G. (1998). Long-distance radionuclide transfer in the Arctic Seas related to fish migrations. Radiation Protection Dosimetry. 75, 219-222.

[12] Coughtrey, P.J. and Thorne, M.C. Radionuclide distribution and transport in terrestrial and aquatic Ecosystems - A critical review of data. Volume 1. A.A. (Balkema: Rotterdam, 1983) pp. 496.

[13] USDoE. A graded approach for evaluating radiation doses to aquatic and terrestrial biota. U.S. Department of Energy. Technical Standard DOE-STD-1153-2002. (USDoE, Washington D.C., U.S.A., 2002).

[14] Higley, K.A., Domotor, S.L. \& Antonio, E.J., Journal of Environmental Radioactivity, 66 (2003), 61-74.

[15] Dommasnes, A, Christensen, V.,Ellertsen, B., Kvamme, C., Melle, W., Nøttestad, L., Pedersen, T., Tjelmeland, \& S., Zeller, D. An ECOPATH model for the Norwegian Sea and Barents Sea. 213-240. In: Fisheries Impacts on North Atlantic Ecosystems: Models and Analyses. Guénette, S., Christensen, V. \& Pauly, D. (Eds.), (Fisheries Centre Research Reports, 9, 4. The Fisheries Centre, Univeristy of British Columbia, Vancouver, Canada, 2001).

[16] Brown, J., Børretzen, P., Dowdall, M., Sazykina, T. \& Kryshev, I. ARCTIC (in press).

[17] Thomann, R.V., Canadian Journal of Fishing and Aquatic Science. 38 (1981), 280-296.

[18] Landrum, P.F., Lee, H., Lydy, M.J., Annual Review: Toxicokinetics in aquatic systems: model comparison and use in hazard assessment. Environmental Toxicology Chemistry, 11 (1992), 1709-1725. 